\title{
Leitlinien - vom lästigen Übel zur Sensation
}

\author{
Prof. Dr. Rainhild Schäfers, Hebamme, Professorin für Hebammenwissenschaft, Bochum
}

\section{Die Kaiserschnittraten in Deutschland stagnieren - allerdings auf einem hohen Niveau. Gleichzeitig werden die Diskussionen um Kaiserschnitte und vaginale Geburten immer lauter. Doch die Regelungen in den existierenden S1-Leitlinien sind weniger eine Hilfe, sondern vielmehr Handlungsempfehlungen, die enormen Interpretationsspielraum zulassen. Das Bundesministerium für Gesundheit hat diese Problematik erkannt und fördert die Entwicklung gleich zweier Leitlinien auf höchstem Entwicklungsniveau.}

\section{Deutsche Kaiserschnitt- raten im internationalen Vergleich}

Wie hoch ist zu hoch? Die optimale Kaiserschnittrate beschäftigt uns nicht nur hierzulande, sondern wird auch international thematisiert [19]. 2010 lag Deutschland im europäischen Vergleich mit seinen Kaiserschnittraten im oberen Drittel. Zypern führte die Liste mit 52,2\% deutlich vor Italien (38,0\%), Rumänien (36,9\%) und Portugal (36,3\%) an, während die Niederlande (17,0\%) sowie nahezu alle skandinavischen Länder unter $20 \%$ lagen [10]. Wenngleich die Kaiserschnittraten in Deutschland seit dem Jahr 2011 nicht mehr steigen, befinden sie sich mit einer Rate von insgesamt $32,2 \%$ [6] dennoch auf einem hohen Niveau.

\section{Morbidität und Mortalität}

Nicht jede Ärztin/jeder Arzt oder Hebamme empfindet die derzeitigen Raten zu hoch und fordert gleichsam den Beweis, dass eine hohe Kaiserschnittrate mit einer höheren Rate mütterlicher wie kindlicher Morbidität und Mortalität einhergeht. Diese Ärztinnen und Hebammen bewerten den Kaiserschnitt als Geburtsmodus, der im Zuge des Selbst- bestimmungsrechtes der Frau frei wählbar sein sollte.

Den Beweis, dass eine hohe Kaiserschnittrate tatsächlich auch die mütterlichen und kindlichen Morbiditäts- und Mortalitätsraten erhöht, muss man derzeit weitestgehend schuldig bleiben. Bislang gibt es nur wenige Studien, die sich mit diesem Aspekt beschäftigen. Überwiegend handelt es sich dabei um retrospektive Datenanalysen, die je nach Auswertungsstrategien nur sehr bedingt die Formulierung eines Kausalzusammenhangs zulassen. Nachweisen lassen sich lediglich statistische Zusammenhänge wie eine erhöhte Rate an Asthma oder Diabetes bei Kindern, die durch einen Kaiserschnitt ohne vorherige Wehentätigkeit geboren wurden [17]. Als gesichert darf die Erkenntnis gelten, dass eine Sectiorate über 15\% keinen Einfluss auf die mütterliche und neonatale Mortalität hat $[19,20]$. Die mangelnden Beweise dürfen jedoch nicht dazu führen, die hohen Kaiserschnittraten zu ignorieren. Vielmehr müssen sie die Bestrebung, die Raten zu senken, noch bestärken. Denn eine für mindestens zwei Menschen bedeutsame Intervention durchzuführen - ohne medizinische Notwendigkeit, dafür aber mit der Unkenntnis über mittel- und langfristige Folgen - ist streng genommen unethisch.

\section{Gründe für einen Kaiserschnitt}

Oftmals wird das Alter der Frauen als eine Ursache der hohen Kaiserschnittraten angeführt. 2015 lag dieses bei der Geburt des 1. Kindes bei durchschnittlich 29,6 Jahren [15]. Die Unterschiede in den Raten sowohl auf Landesebene als auch auf Kreisebene zeigen allerdings, dass nicht allein das höhere Alter der Mutter für die Kaiserschnittraten verantwortlich gemacht werden kann. 2014 erwies sich beispielsweise das Saarland mit einer Rate von $40,2 \%$ bei einem mütterlichen Durchschnittsalter von 29,1 Jahren bei der Geburt des 1. Kindes als das Bundesland mit der höchsten Kaiserschnittrate im Gegensatz zu Sachsen, das mit $24,2 \%$ die geringste Kaiserschnittrate bei einem mütterlichen Durchschnittsalter von 28,9 Jahren bei der Geburt des 1. Kindes aufweist [16]. Auf Kreisebene (Wohnort der Mutter) sind die Unterschiede noch eindrucksvoller und reichen von $17 \%$ in Dresden bis $51 \%$ im Kreis Landau [8].

Die Ursachen für diese Unterschiede bleiben unklar. Möglicherweise liegen sie in der Philosophie eines Krankenhauses oder in der Person der jeweils diensthabenden Ärztin bzw. des diensthabenden Arztes begründet. DeMott und Sandmire 
$[1,2]$ führen an, dass sich innerhalb einer geburtshilflichen Einrichtung die Kaiserschnittraten je nach diensthabender Ärztin/diensthabendem Arzt deutlich unterscheiden. Kalish und McCullough [7] bestätigen dies und stellen zudem fest, dass das Alter, die Art der Spezialisierung sowie die Tätigkeit in Vollzeit der Ärztinnen und Ärzte einen größeren Einfluss auf die Kaiserschnittrate zu haben scheinen als die individuellen Voraussetzungen der Schwangeren. Auch das Geschlecht der behandelnden Person spielt in der Wahl des Geburtsmodus eine Rolle $[5,11]$. Neben diesen personalen Faktoren zeigt sich außerdem, dass der Wochentag [9] sowie die Tageszeit an sich $[14,18]$ mit der Rate der sekundären Kaiserschnitte in Zusammenhang stehen. Es sind also eine Reihe von Faktoren zu identifizieren, die den Kaiserschnitt als Geburtsmodus außerhalb jeglicher medizinischer Notwendigkeit zu begünstigen scheinen.

\section{Bedeutung von Leitlinien fürs geburtshilfliche Prozedere}

Vor diesem Hintergrund und mit Blick auf die möglicherweise nachhaltigen gesundheitlichen Folgen für Mutter und Kind scheint es umso dringender, das geburtshilfliche Prozedere sowohl um die vaginale Geburt als auch um einen Kaiserschnitt in Form einer medizinischen Leitlinie zu definieren.

\section{S1-Leitlinie}

Dabei ist die Entwicklungsstufe, auf der diese Leitlinie entsteht, von besonderer Bedeutung. Der überwiegende Teil der zurzeit existierenden geburtshilflichen Leitlinien sind der niedrigsten Entwicklungsstufe S1 zuzuordnen, was dem Niveau einer Handlungsempfehlung durch Expertinnen entspricht. Dieses Niveau lässt in der Praxis viel Interpretationsspielraum zu.
Manche Leitlinien, wie die S1-Leitlinie zum Vorgehen bei Terminüberschreitung und Übertragung [3] verändern scheinbar über Nacht das geburtshilfliche Management in einzelnen Einrichtungen, während andere, wie die S1-Leitlinie zur strukturellen Voraussetzung der perinatologischen Versorgung in Deutschland [4], kaum Beachtung finden. Letzteres ist aus Hebammensicht besonders bedauerlich, denn in der Leitlinie ist folgende Formulierung zu finden: „Es soll eine kontinuierliche Betreuung jeder Schwangeren sub partu durch eine Hebamme gewährleistet sein. (...) Abhängig von der jährlichen Geburtenrate sollen so viele Hebammen in der Klinik anwesend bzw. rufbereit sein, dass zu mehr als $95 \%$ der Zeit eine Eins zu Eins Betreuung der Gebärenden gewährleistet ist" [4].

\section{S3-Leitlinie}

Die Leitlinienerstellung in Deutschland wurde bislang hauptsächlich durch das ehrenamtliche Engagement der Mitglieder einzelner wissenschaftlich medizinischer Fachgesellschaften getragen. Finanzielle Ressourcen für die Entwicklung einer Leitlinie stehen den Fachgesellschaften nur sehr begrenzt zur Verfügung. Diese wären jedoch nötig, um eine Leitlinie auf der Entwicklungsstufe $\mathbf{S 3}$ zu erstellen oder eine S1-Leitlinie auf das Niveau einer S3-Leitlinie anzuheben.

- Leitlinien auf dem S3-Niveau haben den Anspruch, dass sie von einer Entwicklergruppe erstellt werden, die für den Anwenderkreis repräsentativ ist. Das bedeutet, dass alle Berufsgruppen, die in die Versorgung von Frauen während der Lebensphase Schwangerschaft, Geburt, Wochenbett und Stillzeit involviert sind, sowie Frauenvertreterinnen zur Entwicklergruppe gehören.

- Ein weiteres Qualitätskriterium auf S3-Niveau ist die systematische Evidenzbasierung, die der Leitlinie zugrunde liegt. Hier reicht es nicht, einfach nur in den gängigen Datenbanken nach Studien zu schauen. Eine systematische Evidenzbasierung unterliegt sehr strengen Kriterien und sollte von einem unabhängigen Institut durchgeführt werden.

- Als drittes und letztes Kriterium muss eine S3-Leitlinie in einem strukturierten Konsensverfahren entwickelt werden. Das heißt unter anderem, dass sich die Entwicklergruppe in der Finalisierung der Leitlinie in einem strukturierten Abstimmungsprozess über den Empfehlungsgrad jeder einzelnen Empfehlung, die in einer Leitlinie ausgesprochen wird, verständigt (Soll-, Sollte- oder Kann-Empfehlung).

Für die Erstellung einer S3-Leitlinie wird ein finanzielles Aufkommen in Höhe von 250.000 Euro geschätzt. Um zu verhindern, dass mit der Erstellung privatwirtschaftliche Interessen verfolgt werden, ist die Bereitstellung der Gelder durch unabhängige wissenschaftliche Institutionen oder aus öffentlichen Fonds notwendig. Bislang gibt es hierzu keine etablierten Verfahren. Und so darf es als absolut bemerkenswert angesehen werden, dass das Bundesministerium für Gesundheit (BMG) Ende 2014 vor dem Hintergrund immer lauter werdender Diskussionen um die Kaiserschnittraten in Deutschland, die Bereitstellung von Geldern für die Erstellung gleich zweier S3-Leitlinien (S3-Leitlinie Kaiserschnitt und S3-Leitlinie vaginale Geburt am Termin) in Aussicht gestellt hat.

\section{S3-Leitlinie Kaiserschnitt und S3-Leitlinie vaginale Geburt}

Die Deutsche Gesellschaft für Gynäkologie und Geburtshilfe (DGGG), namentlich Prof. Dr. Dr. hc Frank Louwen, sorgte unmittelbar nach dem Signal aus dem BMG für die Konstituierung eines Koordinatorenteams. Die Teams beider Leitlinien bestehen aus Professorinnen und Professoren der Medizin und der Hebammenwissenschaft. 
Die Zusammenarbeit von Ärztinnen/

Ärzten und Hebammen auf dieser Ebene darf als absoluter Meilenstein in der Leitlinienentwicklung und damit in der Qualitätsentwicklung im deutschsprachigen Raum angesehen werden.

\section{Die Leitliniengruppen}

Das Koordinatorenteam ist verantwortlich für die Bildung der Leitliniengruppen und agiert gleichzeitig als Teil von ihnen. Diese Leitliniengruppen, die letztendlich für die Erstellung der Leitlinien zuständig sind, bestehen aus Vertreterinnen und Vertretern unterschiedlicher Fachgesellschaften und Berufsverbände aus Deutschland, Österreich und der Schweiz sowie Methodikerinnen bzw. Methodikern und Frauenvertreterinnen. Alle Mitglieder der Leitliniengruppen müssen zuvor mögliche Interessenskonflikte offenlegen und sind von Beginn an ehrenamtlich tätig. Eine der ersten Aufgaben der Leitliniengruppen ist die Suche nach und die Bewertung von internationalen Leitlinien zum jeweiligen Thema.

\section{Bewertung internationaler Leitlinien}

Im internationalen Kontext stechen in diesem Zusammenhang die Guidelines des National Institute for Health and Clinical Excellence (NICE) in Großbritannien aufgrund ihres hohen Anspruchs an Evidenzbasierung und Qualität hervor. In welcher Form Inhalte und Empfehlungen dieser Leitlinien bei der Erstellung der Deutschen S3-Leitlinie Berücksichtigung finden, ist derzeit noch unklar (Stand Dezember 2016). Bedacht werden muss dabei, dass die Inhalte von Guidelines immer auch die strukturellen Gegebenheiten des Landes berücksichtigen, für das sie bestimmt sind.

Ein Blick in die NICE Guidelines zeigt, dass eine Orientierung an diesen Guidelines spannende Diskussionen mit sich bringen würde. So heißt es beispielswei- se in der NICE Guideline Cesaerean Section (CS) im Kapitel Indikationen für eine geplante Sectio zur Wahl des Geburtsmodus bei Beckenendlage: „Pregnant women with a singleton breech presentation at term, for whom external cephalic version is contraindicated or has been unsuccessful, should be offered CS because it reduces perinatal mortality and neonatal morbidity“ [12]. Hierzulande sind immer mehr Ärztinnen, Ärzte und Hebammen sehr bemüht, dass sich die vaginale Geburt als Geburtsmodus bei einer Beckenendlage nach Abklärung aller Risiken etabliert. Die Übernahme der obenstehenden Formulierung aus den NICE Guidelines würde diese Bemühungen sicherlich konterkarieren.

Und auch ein Beispiel aus der NICE Guideline Intrapartum Care zeigt zukünftiges Diskussionspotenzial: „Do not perform cardiotocography for low-risk women in established labour (...) If continuous cardiotocography has been used because of concerns arising from intermittent auscultation but there are no non-reassuring or abnormal features (...) on the cardiotocograph trace after 20 minutes, remove the cardiotocograph and return to intermittent auscultation“ [13]. Das birgt Diskussionspotenzial gerade auch vor dem Hintergrund der zur Verfügung stehenden klinisch tätigen Hebammen. Denn längst hat sich das CTG im geburtshilflichen Alltag als Betreuungsersatz für die Hebammen und damit als Ausgleich des Hebammenmangels etabliert. Ein regelmäßiges Auskultieren wäre gleichbedeutend mit einer Eins-zu-Eins-Betreuung und würde zwangsläufig einen höheren Personalschlüssel erfordern.

\section{Chancen von S3-Leitlinien}

Gerade hier zeigt sich das Potenzial einer S3-Leitlinie. Ihre Aufgabe ist nicht, den Status Quo zu bedienen (nicht genügend Hebammen für eine Auskultation im Dienst, ergo: Formulierung der NICE Guidelines ignorieren). Vielmehr soll sie entsprechend der bestmöglichen Eviden- zen Empfehlungen aussprechen (Weniger Interventionen bei Auskultation und Eins-zu-Eins-Betreuung bei gleichem fetalen Outcome, ergo: Personalschlüssel anpassen).

\section{Fazit}

Das wichtigste Argument in der Gesundheitspolitik für die Bereitstellung finanzieller Ressourcen ist die bestmögliche medizinische Versorgung der Bevölkerung. Eine S3-Leitlinie definiert diese bestmögliche Versorgung. Sie kann somit als Argumentationsgrundlage für die Aushandlung von Ressourcen dienen, die zur Bereitstellung der bestmöglichen Versorgung nötig sind. Gleichzeitig bietet sie die Chance eines gelungenen TheoriePraxistransfers, da durch die Aufbereitung der Studienlage der Praxis eine gute Übersicht der aktuellen externen Evidenzen zur Verfügung gestellt wird.

Die eigentliche Sensation ist aber, dass wissenschaftlich tätige Ärztinnen, Ärzte und Hebammen sich gemeinsam für die Erstellung dieser Leitlinien unter Einbezug der Nutzerinnenperspektive verantwortlich fühlen. Sie tun das wohlwissend, dass diese Form der Zusammenarbeit in der jeweils eigenen Berufsgruppe nicht von allen gleichermaßen wohlwollend betrachtet wird.

\section{Literatur}

1 DeMott RK, Sandmire HF. The Green Bay cesarean section study. I. The physician factor as a determinant of cesarean birth rates. Am J Obstet Gynecol 1990; 162 (6): 1593-1599

2 DeMott RK, Sandmire HF. The Green Bay cesarean section study. II. The physician factor as a determinant of cesarean birth rates for failed labor. Am J Obstet Gynecol 1992; 166 (6 Pt 1): 1799-1806

3 Deutsche Gesellschaft für Gynäkologie und Geburtshilfe. Vorgehen bei Terminüberschreitung und Übertragung. Frankfurt: AWMF; 2014

4 Deutsche Gesellschaft für Perinatale Medizin. Empfehlungen für die 
strukturellen Voraussetzungen der perinatologischen Versorgung in Deutschland. Frankfurt: AWMF; 2015

5 Ghetti C, Chan BKS, Guise J-M. Physicians' responses to patient-requested cesarean delivery. Birth 2004; 31 (4): 280-284

6 IQTiG - Institut für Qualität und Transparenz im Gesundheitswesen. (2016). Bundesauswertung zum Erfassungsjahr 2015 Geburtshilfe. Berlin: IQTiG

7 Kalish RB, McCullough L, Gupta M, Thaler HT, Chervenak FA. Intrapartum elective cesarean delivery: a previously unrecognized clinical entity. Obstetrics and gynecology 2004; 103 (6): 1137-1141

8 Kolip P, Nolting H-D, Zich K (2012). Faktencheck Gesundheit - Kaiserschnittgeburten - Entwicklung und regionale Verteilung. Gütersloh: Bertelsmann-Stiftung

$9 \quad$ Lerchl A. Where are the Sunday babies? III. Caesarean sections, decreased weekend births, and midwife involvement in Germany. Naturwissenschaften 2007 (1-6): DOI: 10.1007/s00114-0070306-3

10 Macfarlane AJ, Blondel B, Mohangoo AD, Cuttini M, Nijhuis J, Novak Z, Olafsdottir HS, Zeitlin J. Wide differences in mode of delivery within Europe: risk-stratified analyses of aggregated routine data from the Euro-Peristat study. BJOG : an international journal of obstetrics and gynaecology 2016; 123 (4): 559-568
11 Mitler LK, Rizzo JA, Horwitz SM. Physician gender and cesarean sections. Journal of clinical epidemiology 2000; 53 (10): 1030-1035

12 National Collaborating Centre for Women's and Children's Health. Caesarean section. November 2011. London: National Institute for Health and Clinical Excellence; 2012

13 National Collaborating Centre for Women's and Children's Health. Intrapartum care: care of healthy women and their babies during childbirth - Verison 2. London: National Institute for Health and Clinical Excellence; 2014

14 Spetz J, Smith MW, Ennis SF. Physician incentives and the timing of cesarean sections: evidence from California. Medical care 2001; 39 (6): 536-550

15 Statistisches Bundesamt. Durchschnittliches Alter der Mutter bei der Geburt des Kindes 2015. https://www.destatis. de/DE/ZahlenFakten/GesellschaftStaat/ Bevoelkerung/Geburten/Tabellen/ GeburtenMutterAlterBundeslaender. htmlZugriff am: 14.12.2016

16 Statistisches Bundesamt. Mehr Krankenhausentbindungen 2014 bei gleicher Kaiserschnittrate. Pressemitteilung vom 14. September 2015 - 338/15 https:// www.destatis.de/DE/PresseService/ Presse/Pressemitteilungen/2015/09/ PD15_338_231pdf.pdf;jsessionid=00DBE FB650C4A5ADD6441EE609FCD8F3. cae3?__blob=publicationFile. 14.12.2016

17 Thavagnanam S, Fleming J, Bromley A, Shields MD., Cardwell CR. A meta-analysis of the association between Caesarean section and childhood asthma. Clin Exp Allergy 2008; 38 (4): 629-633
18 Webb DA, Culhane J. Time of day variation in rates of obstetric intervention to assist in vaginal delivery. Journal of epidemiology and community health 2002; 56 (8): 577-578

19 Ye J, Betran AP, Guerrero Vela M, Souza JP, Zhang J. Searching for the optimal rate of medically necessary cesarean delivery. Birth 2014; 41 (3): 237-244. doi: 10.1111/birt.12104

20 Ye J, Zhang J, Mikolajczyk R, Torloni MR, Gülmezoglu AM, Betràn AP. Association between rates of caesarean section and maternal and neonatal mortality in the 21st century: a worldwide populationbased ecological study with longitudinal data.

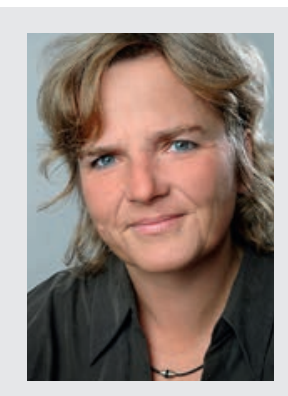

Prof. Dr. Rainhild Schäfers

Professorin für Hebammenwissenschaft

Hochschule für Gesundheit Bochum Gesundheitscampus 6-8 44801 Bochum Rainhild.schaefers@hs-gesundheit.de 